\title{
Effect of Docosahexaenoic Acid Supplementation on Microbiota in Obese ChiLdrEn: A Pilot Study. (The DAMOCLE Study) ${ }^{+}$
}

\author{
Matilde Amatruda ${ }^{1}$, Sara Vizzuso ${ }^{1}$, Valentina Fabiano ${ }^{2}$, Elisa Borghi ${ }^{1}$, Gian VIncenzo Zuccotti ${ }^{2}$ \\ and Elvira Verduci ${ }^{2, *}$ \\ 1 Department of Health Sciences, University of Milan, Milan, Italy; matilde.amatruda@unimi.it (M.A.); \\ sara.vizzuso@unimi.it (S.V.); elisa.borghi@unimi.it (E.B.) \\ 2 Department of Pediatrics, Vittore Buzzi Children's Hospital, University of Milan, Milan, Italy; \\ valentina.fabiano@unimi.it (V.F.); gianvincenzo.zuccotti@unimi.it (G.V.Z.) \\ * Correspondence: elvira.verduci@unimi.it \\ + Presented at the 1st International Electronic Conference on Nutrients - Nutritional and Microbiota Effects \\ on Chronic Disease, 2-15 November 2020; Available online: https://iecn2020.sciforum.net/. \\ Published: 30 October 2020
}

\begin{abstract}
Introduction: Obesity is an inflammatory condition associated with metabolic alterations. Recent researches suggested that gut microbiota plays a role in its pathogenesis, as obesity has been associated with lower bacterial diversity and higher Firmicutes/Bacteroidetes ratio ( $\mathrm{F} / \mathrm{R}$ ratio) compared to normal-weight condition. Such alterations can affect metabolite production (mainly short-chain fatty acids) having an impact on inflammatory markers modulation and insulin secretion. Objectives and study: To determine the effect of 4 months $500 \mathrm{mg} /$ day DHA supplementation, combined with dietary and lifestyle intervention, on gut microbiota and biochemical parameters. Methods: Twelve Caucasian obese children (5 males, 7 females), aged 6-14 years (mean age $10 \mathrm{y}$ ), were enrolled. Study protocol was approved by the local Ethics Commitee and registered on ClinicalTrials.gov (identifier: NCT04151758). Blood and stool samples were collected at baseline (t0), after 4 months DHA supplementation combined with diet-lifestyle intervention ( $\mathrm{t} 1$ ), and after additional 4 months of dietary dietary-lifestyle intervention without DHA supplementation (t2). Anthropometric measures (BMI z score, body circumferences and skinfolds), body composition estimation using air displacement plethysmography system (BOD POD), and 3-day food record were assessed. Biochemical analysis included: glucose metabolism, lipid profile, liver function and inflammatory markers. Insulin sensitivity and insulin resistance were assessed by HOMA index (Homeostasis Model Assessment) and QUICKI (Quantitative Insulin-Sensitivity Check Index), respectively, while cardiovascular risk was estimated trough AIP (Atherogenix Index of Plasma). Gut microbiota analysis was performed by next-generation sequencing using V3-V4 hypervariable 16S rRNA genomic region (Illumina). Results: So far 12 children completed the DHA plus dietary-lifestyle intervention (t0-t1-t2) and 3, out of these 12, completed the entire observation period ( $\mathrm{t} 0-\mathrm{t} 1-\mathrm{t} 2)$. In both groups no longitudinal changes in dietary habits, as well as in BMI z-score, waist circumference and fat mass percentage (FM\%) were recorded. At baseline, most patients showed increased values of HOMA-IR (Mean value 3.30, SD 1.92), AIP (0.24, SD 0.22), VES (26.75 mm/h, SD 12.4) and CPR ( $6.80 \mathrm{mg} / \mathrm{dl}$, SD 2.97), suggesting different grades of insulin resistance, dyslipidemia and systemic inflammation. No significant improvement was found after the intervention ( $\mathrm{t} 1$ ). Alanine transferase values were slightly reduced from t0 ( 34.33 , $\mathrm{SD} 5.69 \mathrm{IU} / \mathrm{L})$ to $\mathrm{t} 1(31.33, \mathrm{SD} 11.68 \mathrm{IU} / \mathrm{L})$ and difference became statistically significant from t0 to 2 $(p=0.014,3$ subjects). While alpha-diversity analysis revealed no significant differences in gut microbiota biodiversity at different time-points, phylogenetic analysis highlighted a significant separation of $\mathrm{t} 0$ and $\mathrm{t} 1$ bacterial communities according to weighted Unifrac distances $(p=0.017)$. In particular, a significant lowering of F/R ratio from t0 (4.04) to t1 (2.20) was observed. Conclusions: Lowering of $\mathrm{F} / \mathrm{R}$ ratio from $\mathrm{t} 0$ to $\mathrm{t} 1$ seems to be associated exclusively to DHA supplementation. The
\end{abstract}


study is still on-going and more data from $\mathrm{t} 2$ time-point, as well as microbial metabolite analysis, are needed to support the potential impact of DHA supplementation.

Keywords: gut microbiota; DHA, childhood obesity

\section{Introduction}

The global prevalence of obesity has shown an alarming rise in the last 4 decades, assuming epidemic proportions [1]. According to World Health Organization (WHO) data in 2016 almost 39\% of global population was overweight and $13 \%$ of the world's adult population was obese. Among children and adolescents aged 5-19 years, over 340 million were overweight or obese. In 2019 approximately 38.2 million children under the age of 5 years were overweight or obese [2].

Obesity is an inflammatory condition associated with several metabolic alterations including central adiposity, elevated blood pressure, dyslipidemia and abnormal glucose metabolism which tend to cluster in the metabolic syndrome [3]. Increasing interest in pediatric obesity has been focused on identification of screening tools for metabolic complications.

HOmeostasis Model Assessment of insulin resistance (IR) index (HOMA-IR) and QUantitative Insulin-sensitivity ChecK Index (QUICK index) calculated on fasting samples are useful tools used in the clinical practice to identify subjects at risk for type 2 diabetes mellitus [4]. Atherogenic index of plasma (AIP) reflects the relationship between protective and atherogenic lipoproteins and may predict the risk of cardiovascular diseases in adults and adolescents [5] and is emerging as an useful tool for detecting metabolic syndrome in children and adolescents [6].

It's well known that dietary pattern can affect gut microbiome composition, but recent researches suggested that gut microbiota could play a role in the pathogenesis of the disease and metabolic complications [7]. A high-fat and high-sugar "Western-style" diet increases the relative abundance of Firmicutes at the expense of Bacteroidetes has been observed in animal models [8] and also in humans [9].

As been associated with lower bacterial diversity and higher Firmicutes/Bacteroidetes ratio (F/R ratio) compared to normal-weight condition. Such alterations can affect metabolite production (mainly short-chain fatty acids) having an impact on inflammatory markers modulation and insulin secretion [10].

Polyunsaturated Fatty Acids (LCPUFAs) are known for their health benefits, especially in relation to their ability to modulate inflammation and improve some obesity-associated comorbidities [11]. Recent research is focusing on the potential impatct of LCPUFAs, especially Docosahexaenoic acid (DHA), supplementation on anthropometric and metabolic parameters in obese children [12].

\section{Objectives and Study}

The aim of our study was to determine the effect of 4 months $500 \mathrm{mg} /$ day DHA supplementation, combined with dietary intervention and lifestyle education, on gut microbiota and metabolic biochemical parameters in a group of obese Caucasian children attending our Clinical Nutrition Service.

\section{Methods}

Twelve Caucasian obese children (5 males, 7 females), aged 6-14 years (mean age $10 \mathrm{y}$ ), were enrolled from November 2018 to October 2019 at our Pediatric Department. Study protocol was approved by the local Ethics Commitee and registered on ClinicalTrials.gov (identifier: NCT04151758).

Blood and stool samples were collected at baseline ( $\mathrm{t} 0)$, after 4 months of DHA supplementation combined with diet-lifestyle intervention (t1), and lastly after additional 4 months of dietary dietarylifestyle intervention without DHA supplementation (t2). Blood samples were analyzed to check 
metabolic assessment that included: total cholesterol (TC), HDL-c, low-density lipoprotein cholesterol (LDL-c), TG, insulin, fasting glucose, glycated hemoglobin (HbA1c). Insulin sensitivity and insulin resistance were assessed by HOMA index (Homeostasis Model Assessment) and QUICKI (Quantitative Insulin-Sensitivity Check Index), while cardiovascular risk was estimated trough AIP (Athrtogenix Index of Plasma). Gut microbiota analysis was performed by next-generation sequencing using V3-V4 hypervariable $16 \mathrm{~S}$ rRNA genomic region (Illumina).

Anthropometric measures (BMI z-score, body circumferences and skinfolds), body composition estimation using air displacement plethysmography system (BOD POD) were also assessed ( $\mathrm{t} 0-\mathrm{t} 1-$ t2). Body weight and height were measured using a mechanical column scale (seca 711; seca GmbH \& KG, Hamburg, Germany) with an integrated measuring rod (seca 220; seca GmbH \& KG, Hamburg, Germany). Body mass index (BMI) was calculated as weight $/ \mathrm{height}^{2}\left(\mathrm{~kg} / \mathrm{m}^{2}\right)$. BMI $z$-score was assessed according to WHO curves specific for age and sex. Waist circumference (WC) was measured trough seca measuring tape 203 (seca $\mathrm{GmbH} \& \mathrm{KG}$ ) at midpoint between costal margin and iliac crest, in a standing position, at the end of a gentle expiration. Tricipital skinfold thickness was measured on the left side of the body, using a Harpenden Skinfold plicometer (Chasmors Ltd., London, UK) at the midpoint between acromion process and olecranon process.

Fat mass (FM), FM percentage (FM\%), fat-free mass (FFM) and fat-free mass percentage (FFM\%) were estimated using a bioelectrical impedance analysis system (BC 418 MA, Tanita Corp, Tokyo, Japan). An oscillometric device was used to check blood pressure (BP), according to the National High Blood Pressure Education Program Working Group recommendations. To assess dietary habits and food intake a 3-day food record, filled out by parents with support of trained dietitian, was used. Usual portion sizes were estimated using household measures and the weight (e.g., pasta) or unit (e.g., fruit juice) of the purchase.

\section{Results}

So far, 9 children attended the first control ( $\mathrm{t} 0-\mathrm{t} 1)$ and 3 of them completed the entire observation period ( $\mathrm{t} 0-\mathrm{t} 1-\mathrm{t} 2)$. In both groups no changes in dietary habits, as well as in body mass index (BMI) zscore, waist circumference and fat mass percentage (FM\%) were recorded. At baseline, most patients showed increased values of HOMA-IR (Mean value 3.30, SD 1.92), AIP (0.24, SD 0.22), Erythrocyte Sedimentation Rate (ESR) (26.75 mm/h, SD 12.4) and C-Reactive Protein (CRP) (6.80 mg/dl, SD 2.97), suggesting different grades of insulin resistance, dyslipidemia and systemic inflammation. No significant improvement was found after the intervention. Alanine aminotransferase (ALT) values were slightly reduced from t0 $(34.33$, SD 5.69$)$ to $11(31.33$, SD 11.68) and difference became statistically significant from $\mathrm{t} 0$ to $\mathrm{t} 2$ ( $p=0.014,3$ subjects). While alpha-diversity analysis revealed no significant differences in gut microbiota biodiversity at different time-points, phylogenetic analysis highlighted a significant separation of $\mathrm{t} 0$ and $\mathrm{t} 1$ bacterial communities according to weighted Unifrac distances $(p=0.017)$. In particular, a significant lowering of $\mathrm{F} / \mathrm{R}$ ratio from t0 $(4.04)$ to $\mathrm{t} 1(2.20)$ was observed.

\section{Conclusions}

Our findings show that lowering of $\mathrm{F} / \mathrm{R}$ ratio from to to $\mathrm{t} 1$ is associated exclusively to DHA supplementation, regardless of dietary and lifestyle intervention impact. The reduction of $\mathrm{F} / \mathrm{R}$ ratio is even more evident at the $\mathrm{t} 2$ control, suggesting a delayed effect after DHA discontinuation. The study is still on-going and more data from $\mathrm{t} 2$ time-point, as well as from microbial metabolite analysis, are needed to support the potential impact of DHA supplementation on gut microbiota and metabolism of obese children. 
(A)

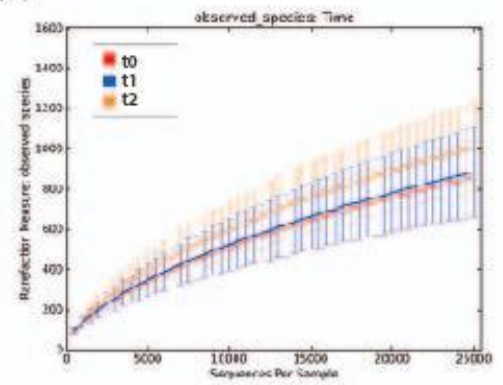

(B)

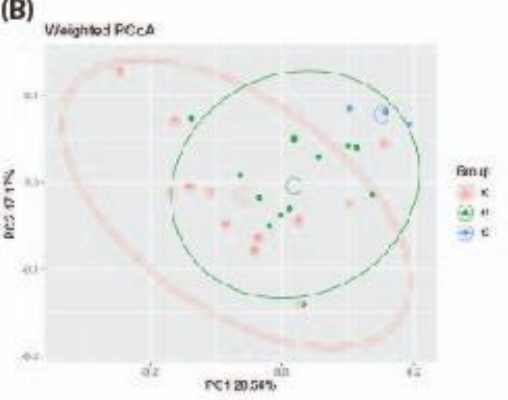

(C)

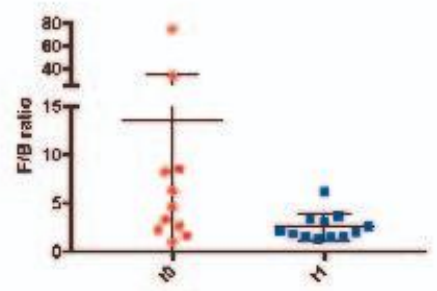

Figure 1. Gut microbiota analysis in obese children baseline (t0), after 4 months DHA supplementation combined with diet-lifestyle intervention (t1), and after additional 4 months of dietary dietary-lifestyle intervention without DHA supplementation (t2). (A) alpha-diversity (observed species metric is reported) showed no significant differences in gut microbiota biodiversity; (B) Principal Coordinate Analysis (PCoA) according to Weighted Unifrac distance: t0 samples (red) significantly $(p<0.05)$ separates from t1 samples (green); (C) Firmicutes/Bacteroidetes (F/B) ratio significantly decreases $(p<0.05)$ at $\mathrm{t} 1$ (blue) compared with t0 (red).

Conflicts of Interest: The authors declare no conflicts of interest.

\section{References}

1. Jaacks, L.M.; Vandevijvere, S.; Pan, A.; McGowan, C.J; Wallace, C.; Imamura, F.; Mozaffarian, D.; Swinburn, B.; Ezzati, M. The obesity transition: Stages of the global epidemic. Lancet Diabetes Endocrinol. 2019, 7, 231240.

2. Available online: https://www.who.int/data/gho.

3. Han, T.S.; Lean, M.E. A clinical perspective of obesity, metabolic syndrome and cardiovascular disease. JRSM Cardiovasc. Dis. 2016, 5, 2048004016633371.

4. Shashaj, B.; Luciano, R.; Contoli, B.; Morino, G.S.; Spreghini, M.R.; Rustico, C.; Sforza, R.W.; Dallapiccola, B. Melania Manco. Reference ranges of HOMA-IR in normal-weight and obese young Caucasians. Acta Diabetol. 2016, 53, 251-260.

5. Niroumand, S.; Khajedaluee, M. Atherogenic Index of Plasma (AIP): A marker of cardiovascular disease. Med. J. Islam. Repub. Iran 2015, 29, 240.

6. $\quad$ Ejtahed, H.S.; Kelishadi, R.; Hasani-Ranjbar, S.; Angoorani, P.; Motlagh, M.E.; Shafiee, G.; Ziaodini, H.; Taheri, M.; Qorbani, M.; Heshmat, R. Discriminatory Ability of Visceral Adiposity Index as an Indicator for Modeling Cardio-Metabolic Risk Factors in Pediatric Population: The CASPIAN-V Study. J. Cardiovasc. Thorac. Res. 2019, 11, 280-286.

7. Davis, C.D. The Gut Microbiome and Its Role in Obesity. Nutr Today 2016, 51, 167-174.

8. Turnbaugh, P.J., Bäckhed, F.; Fulton, L.; Gordon, J.I. Diet-induced obesity is linked to marked but reversible alterations in the mouse distal gut microbiome. Cell Host Microbe 2008, 3, 213-223.

9. Wu, G.D.; Chen, J.; Hoffmann, C.; Bittinger, K.; Chen, Y.Y.; Keilbaugh, S.A.; Bewtra, M.; Knights, D.; Walters, W.A.; Knight, R.; et al. Linking long-term dietary patterns with gut microbial enterotypes. Science 2011, 334, 105-108.

10. Magne, F.; Gotteland, M.; Gauthier, L.; Zazueta, A.; Pesoa, S.; Navarrete, P.; Balamurugan, R. The Firmicutes/Bacteroidetes Ratio: A Relevant Marker of Gut Dysbiosis in Obese Patients? Nutrients 2020, 12, 1474 .

11. Amatruda, M.; Ippolito, G.; Vizzuso, S.; Vizzari, G.; Banderali, G.; Verduci, E. Epigenetic Effects of n-3 LCPUFAs: A Role in Pediatric Metabolic Syndrome. Int. J. Mol. Sci. 2019, 20, 2118.

12. Vasickova, L.; Stavek, P.; Suchanek, P. Possible effect of DHA intake on body weight reduction and lipid metabolism in obese children. Neuro Endocrinol. Lett. 2011, 32 (Suppl. 2), 64-67.

Publisher's Note: MDPI stays neutral with regard to jurisdictional claims in published maps and institutional affiliations. 
(C) 2020 by the authors. Licensee MDPI, Basel, Switzerland. This article is an open access article distributed under the terms and conditions of the Creative Commons Attribution (CC BY) license (http://creativecommons.org/licenses/by/4.0/). 